
internationales

vol. $21-n^{\circ} 3 \mid 2005$

Familles, destins personnels et appartenances collectives en migration

Regards croisés sur l'ex-Yougoslavie. Des chercheurs face à leurs objets de recherche et aux événements sociaux et politiques, Varro Gabrielle (dir.)

L'Harmattan, Espaces culturels, Paris, 2005, 246 p. ISBN : 2747584453

\title{
Thomas Brisson
}

\section{(2) OpenEdition \\ Journals}

Édition électronique

URL : https://journals.openedition.org/remi/4322

DOI : $10.4000 /$ remi.4322

ISSN : $1777-5418$

Éditeur

Université de Poitiers

Édition imprimée

Date de publication : 1 décembre 2005

Pagination : 209-212

ISBN : 2-911627-45-8

ISSN : 0765-0752

Référence électronique

Thomas Brisson, «Regards croisés sur l'ex-Yougoslavie. Des chercheurs face à leurs objets de recherche et aux événements sociaux et politiques, Varro Gabrielle (dir.) », Revue européenne des migrations internationales [En ligne], vol. 21 - $n^{\circ} 3$ | 2005, mis en ligne le 27 novembre 2008, consulté le 14 avril 2022. URL : http://journals.openedition.org/remi/4322 ; DOI : https://doi.org/10.4000/remi.4322

Ce document a été généré automatiquement le 14 avril 2022.

(c) Université de Poitiers 


\title{
Regards croisés sur l'ex-Yougoslavie. Des chercheurs face à leurs objets de recherche et aux événements sociaux et politiques, Varro Gabrielle (dir.)
}

\author{
L'Harmattan, Espaces culturels, Paris, 2005, 246 p. ISBN : 2747584453
}

\section{Thomas Brisson}

\section{RÉFÉRENCE}

Regards croisés sur l'ex-Yougoslavie. Des chercheurs face à leurs objets de recherche et aux événements sociaux et politiques, VARRO Gabrielle (dir.), L'Harmattan, Espaces culturels, Paris, 2005, 246 p. ISBN : 2747584453

1 Les neuf articles regroupés au sein de l'ouvrage coordonné par Gabrielle Varro offrent au lecteur une série d'analyses dont il faut signaler la qualité individuelle tout autant que la richesse et la diversité. Les « regards croisés » à l'œuvre sont tout d'abord ceux de chercheurs d'horizons différents: cinq sont français, un suisse, quatre sont originaires de l'ex-Yougoslavie, l'ouvrage évitant ainsi l'écueil des analyses «du dehors» comme celui du point de vue strictement interne. Sa diversité repose également sur un choix d'objets de recherche variés (les couples mixtes, les langues, les rapports sociaux de sexes, les questions identitaires, les manuels scolaires) qui sont l'occasion d'une approche disciplinaire plurielle empruntant à la sociologie, à l'histoire ou à la linguistique. Ce dernier point est d'autant plus important que, de manière revendiquée, l'ouvrage a été conçu comme une recherche de non-spécialistes. C'est bien souvent parce que l'ex-Yougoslavie faisait question par rapport aux travaux qu'ils menaient sur d'autres sujets que les chercheurs ont ici appliqué leurs compétences disciplinaires au cas yougoslave. 
2 Un même souci anime cependant chacune de ces contributions : la volonté d'articuler les analyses scientifiques à une réflexion politique plus large, souci qui s'ancre dans un effort constant pour penser le rapport entretenu par le chercheur avec ses objets de recherche. L'ouvrage représente ainsi une réussite convaincante d'une tentative aussi risquée qu'attirante pour nombre de chercheurs : penser un objet contemporain dont la dimension intellectuelle est inséparable de questions politiques et éthiques. Une de ses forces est probablement d'avoir su assumer la subjectivité des chercheurs et de l'avoir posée comme une dimension indépassable de l'analyse. C'est peut-être le premier article, celui de $\mathrm{G}$. Varro, qui pousse le plus loin la réflexion dans ce sens, allant jusqu'à adopter un système de double écriture où le point de vue scientifique alterne avec les hésitations de la chercheuse sur son terrain et où l'effort de compréhension s'accompagne d'autant d'hésitations, y compris sur le bien-fondé de ses questions. Travaillant sur la question des couples mixtes, la chercheuse est en effet confrontée à un objet particulièrement mouvant : à la difficulté de trouver les individus en question après dix ans de guerre s'ajoute celle de travailler dans un pays où la mémoire est sans cesse reconstruite (avant la guerre, tout le monde vivait ensemble...) et où l'identité est aujourd'hui prise dans une sorte de double bind qui fait que les mêmes accords ayant entériné la séparation ethnique somment de la combattre. Que veut dire, dans ces conditions, travailler sur des couples mixtes? Les difficultés de recherche dont nous fait part Gabrielle Varro semblent s'ancrer dans la complexité à localiser un fait de métissage sans cautionner pour autant une quelconque différenciation primordiale qui fonderait ce même métissage. Il est intéressant de voir que c'est à ce niveau que la réflexion politique vient au secours des difficultés théoriques: indiquant comment l'exemple yougoslave a modifié sa perception du problème, la chercheuse en vient à avancer l'idée que les couples mixtes doivent être traités « non seulement comme un fait social et sociologique mais (aussi comme) un fait politique ». Si les catégories sont appréhendées comme le fruit de rapports sociaux, les guerres de Yougoslavie montrent à quel point une histoire politique peut à son tour les recomplexifier. La référence finale de l'article à la présidentielle française de 2002 s'inscrit ainsi dans le cadre d'une réflexion scientifique attentive aux effets du politique sur la manière dont les individus trouvent (ou non) des manières de vivre ensemble.

3 L'article de S. Wahnich qui lui fait suite est probablement l'article le plus déroutant de l'ouvrage. S'intéressant aux œuvres d'art qui ont abordé la question du conflit yougoslave, la chercheuse interroge le rapport que construisent ces «œuvres et (ces) lieux culturels à un présent post-historique». L'idée d'étudier les conséquences de l'histoire yougoslave récente à travers les productions artistiques contemporaines est une idée aussi intéressante que féconde tant ces dernières mettent profondément en question notre relation à l'histoire, la Yougoslavie devenant, selon S. Wahnich le lieu "d'une expérience historique de la crise de conscience historique ». L'analyse de films ou d'expositions permet ainsi d'accéder à des points de vue décalés, ironiques, irrévérencieux ou interrogateurs sur le conflit yougoslave, tel les photos de Milomir Kovacevic où les portraits de Tito apparaissent dans divers intérieurs dévastés par la guerre. Reste cependant une difficulté inhérente à l'article, celle d'essayer de traduire par écrit une expérience esthétique qui procède d'autres logiques. Le mode d'exposition choisi, très éclaté, proche de certaines écritures postmodernes, est pleinement justifié par son objet mais pose sûrement autant de problèmes qu'il n'en résout. On aurait aimé plus d'éclaircissement et de 
contextualisation sur les œuvres, quitte à sacrifier parfois à un mode d'exposition plus classique, et ce, d'autant plus que le lecteur n'a pas accès aux œuvres en question.

4 L'article d'U. Habul aborde, lui, un sujet plus classique, celui de «l'identité non reconnue de la Bosnie-Herzegovine ». Son intérêt est évident car on se souvient à quel point la Bosnie a été, par la mixité de ses populations, le lieu des affrontements les plus déchirants du conflit yougoslave. Poser aujourd'hui la question en termes d'identité permet ainsi de revenir sur l'un des points les plus sensibles de cette histoire, tant l'identification des individus à des groupes sociaux ou ethniques pose ici problème. La force de l'article d'U. Habul tient dans la diversité des reconfigurations qu'il opère. Historiquement tout d'abord, en posant radicalement la question d'une spécificité bosniaque et en montrant comment celle-ci s'est construite, et a perduré, par-delà la différence de ses populations. Sociologiquement, ensuite, en montrant l'importance du rôle des citadins dans ce processus, évitant ainsi une vision trop monolithique des habitants de la Bosnie-Herzegovine. Ethnologiquement, enfin, mais de manière un peu moins convaincante, tant on aurait souhaité plus de précisions sur le sujet. L'article se conclut par une analyse particulièrement intéressante de la question des mariages mixtes qui le rapproche de l'article de G. Varro. Montrant comment ces mariages sont loin de constituer à eux seuls une solution à l'avenir de la Bosnie, la chercheuse conclut sur la nécessité d'une interrogation politique plus large, notamment en termes de citoyenneté.

5 Janko Mursak aborde, quant à lui, l'histoire politique de la Slovénie. L'article intéresse en particulier par sa question centrale, celle de la possibilité de construire un État sur un territoire aussi étroit. Les analyses de Mursak tendent en effet à montrer la continuité d'une spécificité slovène sur un espace pourtant réduit et entouré de puissants voisins. Sans le poser explicitement, il inverse, de manière assez convaincante, les analyses classiques sur la relation entre États et Nations. Hobsbawm et Anderson, parmi d'autres, nous ont en effet appris à penser que, loin d'en être une émanation, c'est l'État qui "crée " la Nation. La Slovénie semble montrer qu'une spécificité nationale a précédé, de plusieurs siècles, la création récente d'un État. Point de vue d'autant plus intéressant que Mursak est loin d'esquiver les multiples contradictions et oppositions qui structurent la société slovène. On retiendra également de son article d'intéressantes analyses sur le rôle de Tito ainsi que d'utiles précisions sur la différence entre citoyenneté et nationalité dans le cas yougoslave.

6 Spécialiste des rapports sociaux de sexes, Jacqueline Heinen analyse, dans l'article suivant, la place occupée par les femmes dans le conflit yougoslave. Elle s'intéresse plus particulièrement à la manière dont la variable genre a fonctionné par rapport à la question du pacifisme, de l'ethnicisme et du nationalisme. À l'opposé des vulgates nationalistes qui cantonnent les femmes au simple rôle de mère, elle montre comment celles-ci ont su développer un certain nombre d'initiatives pour promouvoir un dialogue entre les différentes parties qui constituaient la Yougoslavie. Ses références à divers types de mobilisations (rencontres à l'étranger, création de réseaux), sont d'autant plus convaincantes que la chercheuse est loin d'accréditer une vision irénique du rôle joué par les femmes. Elle en montre les limites ainsi que les multiples difficultés à s'abstraire complètement des logiques plus générales du conflit.

7 S'intéressant à la réécriture de l'histoire dans les manuels serbes après 2000, M. Radenkovic articule son analyse à l'idée d'un "nationalisme d'en haut». Ce concept global lui permet de ne pas limiter son propos à une simple mise en 
perspective de textes, mais d'inclure ces derniers dans un ensemble social et politique plus large. On retiendra en particulier l'attention portée par le chercheur aux institutions partie prenante de ce processus, ainsi qu'une analyse fine des trajectoires des historiens chargés de la rédaction des manuels. La référence précise à divers extraits de manuels est, de plus, poursuivie par une analyse des réactions des enseignants serbes aux nouveaux contenus d'enseignement. Le croisement de divers outils d'analyse - entretiens, analyses historiques et textuelles - permet ainsi de ressaisir dans toute sa complexité un moment essentiel de constitution du phénomène national.

8 Renouant avec une problématique de l'identité, l'article d'A. Prohic s'intéresse, lui, aux auto-désignations des citoyens de l'ex-Yougoslavie. À partir d'une question ouverte sur la nationalité que s'attribuent les individus, la chercheuse distingue trois types de désignation. Le premier est fondé sur une appartenance ethnique, le deuxième est fonction du lieu de résidence (toponyme), la troisième forme une catégorie de réponses plus larges et diverses (" cosmopolites ", " sans nationalité » etc.). L'article s'intéresse plus particulièrement à la répartition de ces réponses à l'intérieur de deux groupes d'enquêtés: le groupe in (individus habitant sur le territoire de l'exYougoslavie) et le groupe out (habitant à l'extérieur). C'est dans ce dernier groupe que l'on trouve le plus de réponses évitant la référence aux trois nationalités (serbe, bosniaque, croate). L'auteur analyse ainsi l'effort de création déployé par les individus dans un contexte de changements forts : les catégories, loin d'être figées, sont soumises à une capacité d'invention qui redéfinit les identités.

9 L'article de P. Seriot qui lui fait suite illustre de manière frappante l'imbrication des points de vue savant et politique qui caractérise l'ensemble de l'ouvrage. Croisant analyses linguistique et socio-historique pour retracer l'émergence des différentes langues sur le territoire yougoslave, l'auteur montre comment la langue est une donnée profondément travaillée par le politique. «Être soi, c'est inventer la langue des autres ", écrit-il, montrant alors comment la création de langues officielles répond à des contextes sociaux et politiques précis qui définissent autant de communautés et d'ensembles politiques. On appréciera la lucidité du linguiste face aux tentatives d'utilisation de sa discipline à des fins politiques ou identitaires, et son refus de cautionner les jeux du pouvoir. Tout comme l'article de M. Radenkovic sur les manuels scolaires, les analyses de P. Seriot pointent vers une conception réflexive de l'activité intellectuelle dont la dimension politique s'affirme paradoxalement à l'encontre des politiques.

10 La conclusion de l'ouvrage revient à l'article de C. Dubar dont les travaux menés de longue date sur la question de l'identité rentrent parfaitement en congruence avec les questions posées par le cas de l'ex-Yougoslavie. Retravaillant sa distinction entre conceptions essentialiste et nominaliste de l'identité, C. Dubar en vient à dégager un certain nombre de processus d'identification à l'œuvre dans le cas yougoslave, signalant l'importance du territoire, de la religion, de la nation, de la langue et des expériences. Sa conception nominaliste de l'identité lui permet alors de prendre en compte la pluralité des identifications à l'œuvre en ex-Yougoslavie. Le chercheur enrichit finalement son analyse en resituant ces processus dans une triple logique (historique, linguistique et sociologique), qui réinscrit les trajectoires de populations dans autant de configurations et de rapports au politique et au pouvoir. 
11 Ouvrage pluriel, Regards croisés sur l'ex-Yougoslavie, intéressera ainsi tout autant les spécialistes de la question que les non-spécialistes soucieux de comprendre une histoire récente complexe. En plus de la qualité des contributions déjà signalée, les chercheurs seront sensibles à l'effort permanent déployé dans chacun des articles pour redéfinir l'articulation du scientifique et du politique. Si le cas particulier de l'exYougoslavie rendait difficile de se limiter à une simple analyse scientifique, il n'en reste pas moins que l'ouvrage va plus loin que la vision d'un chercheur en position d'extériorité face à l'histoire. Parce que chacun des chercheurs réunis ici travaille sur des questions qui ont fait l'objet d'une utilisation politique dans un contexte de guerre, tous pointent vers l'importance de mettre en œuvre une conception réflexive des sciences sociales qui puisse extraire ses objets - les langues, les identités, l'histoire des manipulations politiques les plus extrêmes dont l'ex-Yougoslavie a fourni un exemple tragique.

\section{AUTEURS}

THOMAS BRISSON

Laboratoire Printemps, UVSQ 